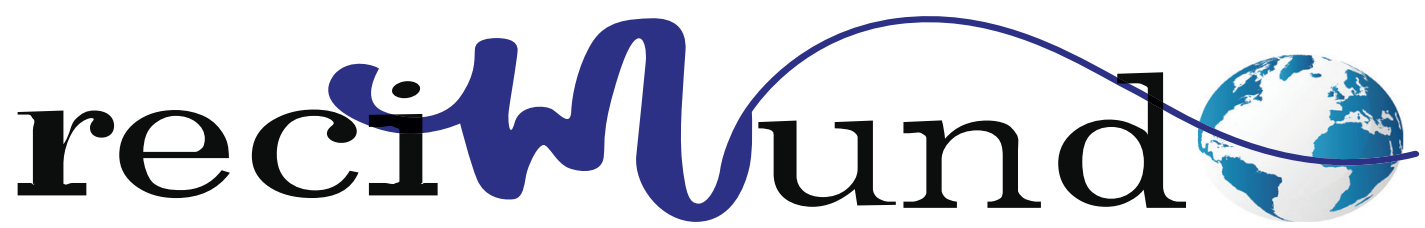

Revista Científica Mundo de la Investigación y el Conocimiento

DOI: 10.26820/recimundo/4.(1).esp.marzo.2020.219-228

URL: http://recimundo.com/index.php/es/article/view/794

EDITORIAL: Saberes del Conocimiento

REVISTA: RECIMUNDO

ISSN: 2588-073X

TIPO DE INVESTIGACIÓN: Artículo de Revisión

CÓDIGO UNESCO: 3201 Ciencias Clínicas

PAGINAS: 219-228

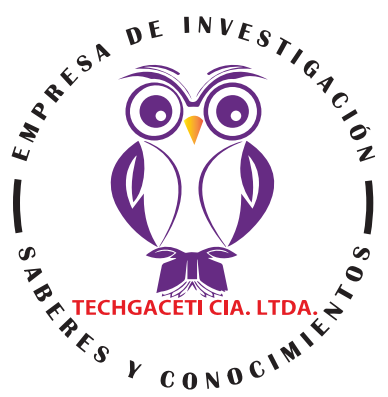

\title{
Gastrostomía endoscópica percutánea indicaciones clínicas
}

\section{Percutaneous endoscopic gastrostomy clinical indications}

Indicações clínicas da gastrostomia endoscópica percutânea

María Verónica Crespo Zamora'; Isis Natali Parrales Vidal2; Genesis Carolina Romoleroux Uquillas;; Jonathan Steven Coello Vergara ${ }^{4}$

RECIBIDO: 20/11/2019 ACEPTADO: 29/01/2020 PUBLICADO: 05/03/2020

1. Médico; Investigador Independiente; Guayaquil, Ecuador; veronicacrespoz29@outlook.com; (iD https://orcid. org/0000-0002-4098-7618

2. Médico; Investigador Independiente; Guayaquil, Ecuador; isisparrales_@hotmail.com; iD https://orcid. org/0000-0002-5235-1350

3. Médico; Investigador Independiente; Guayaquil, Ecuador; gcromolerouxczs5@gmail.com; iD https://orcid. org/0000-0002-7228-1804

4. Médico; Investigador Independiente; Guayaquil, Ecuador; jonathansc@outlook.es; (iD https://orcid.org/00000001-6055-2243

\section{CORRESPONDENCIA}

María Verónica Crespo Zamora

veronicacrespoz29@outlook.com

Guayaquil, Ecuador 


\section{RESUMEN}

Numerosos trastornos menoscaban o disminuyen la capacidad del paciente para tragar pueden beneficiarse de una colocación de la sonda PEG. Esta es considerada la técnica de alimentación electiva si un sistema digestivo funcional está presente. La alimentación PEG ha demostrado consistentemente ser más eficaz y seguro de la alimentación por sonda nasogástrica, después de haber también reemplazado técnicas de gastrostomía quirúrgicos y radiológicos para la alimentación a largo plazo. PEG se considera un procedimiento mínimamente invasivo para asegurar una fuente adecuada para la nutrición enteral en pacientes institucionalizados y en el hogar. Las condiciones crónicas asociadas con riesgo de desnutrición y beneficio disfagia de colocación PEG: trastornos neuro-muscular degenerativos, un cuerpo creciente de evidencia apoya las ventajas de tubos PEG en pacientes con cáncer de cabeza y cuello y en una amplia gama de situaciones en pediátrica. Se discute la seguridad de la colocación PEG bajo medicación antitrombótica. Si bien la profilaxis antibiótica reduce las tasas de infección de la herida periostomal, soluciones de cotrimoxazol administradas a través de un catéter recién insertado constituye una alternativa a los antibióticos intravenosos. La alimentación temprana (3-6 horas) después de la colocación PEG apoya firmemente en evidencias de seguridad, además, resultando en costes reducidos y estancias hospitalarias. Las complicaciones de la PEG son poco frecuentes y la mayoría de los cuidados de enfermería impidieron ser apropiados. La alimentación PEG proporciona el acceso más valioso para la nutrición en pacientes con un sistema gastrointestinal funcional. Su alta eficacia, seguridad y coste reducido subyacen creciente popularidad en todo el mundo.

Palabras clave: Gastrostomía, Alimentación por sonda, Nutrición entera, Tubos de alimentación gástrica, Intubación, Gastrointestinal, Cuidado de enfermera, Complicación.

\section{ABSTRACT}

Many disorders that impair or decrease the patient's ability to swallow may benefit from a PEG tube placement. This is considered the elective feeding technique if a functional digestive system is present. PEG feeding has consistently been shown to be more effective and safer than nasogastric tube feeding, having also replaced surgical and radiological gastrostomy techniques for long-term feeding. PEG is considered a minimally invasive procedure to ensure an adequate source for enteral nutrition in institutionalized patients and at home. Chronic conditions associated with risk of malnutrition and dysphagia benefit from PEG placement: degenerative neuro-muscular disorders, a growing body of evidence supports the advantages of PEG tubes in patients with head and neck cancer and in a wide range of pediatric situations. The safety of PEG placement under antithrombotic medication is discussed. Although antibiotic prophylaxis reduces periostomal wound infection rates, cotrimoxazole solutions administered through a newly inserted catheter is an alternative to intravenous antibiotics. Feeding early (3-6 hours) after PEG placement strongly supports evidence of safety, furthermore, resulting in reduced costs and hospital stays. Complications of PEG are infrequent, and most nursing care prevented them from being appropriate. PEG feeding provides the most valuable access for nutrition in patients with a functional gastrointestinal system. Its high efficiency, safety and low cost underlie increasing popularity worldwide.

Keywords: Gastrostomy, Tube feeding, Enteral nutrition, Gastric feeding tubes, Intubation, Gastrointestinal, Nurse care, Complication.

\section{RESUMO}

Muitos distúrbios que prejudicam ou diminuem a capacidade do paciente de engolir podem se beneficiar da colocação do tubo de PEG. Esta é considerada a técnica de alimentação eletiva se um sistema digestivo funcional estiver presente. A alimentação com PEG tem demonstrado consistentemente ser mais eficaz e mais segura que a alimentação por sonda nasogástrica, tendo também substituído as técnicas de gastrostomia cirúrgica e radiológica para alimentação a longo prazo. O PEG é considerado um procedimento minimamente invasivo para garantir uma fonte adequada de nutrição enteral em pacientes institucionalizados e em casa. As condições crônicas associadas ao risco de desnutrição e disfagia se beneficiam da colocação do PEG: distúrbios neuromusculares degenerativos, um corpo crescente de evidências apóia as vantagens dos tubos de PEG em pacientes com câncer de cabeça e pescoço e em uma ampla variedade de situações pediátricas. A segurança da colocação do PEG sob medicação antitrombótica é discutida. Embora a profilaxia com antibióticos reduza as taxas de infecção periostomal da ferida, as soluções de cotrimoxazol administradas por um cateter recém-inserido são uma alternativa aos antibióticos intravenosos. A alimentação precoce (3-6 horas) após a colocação do PEG suporta fortemente evidências de segurança, além disso, resultando em custos reduzidos e internações hospitalares. As complicações do PEG são raras e a maioria dos cuidados de enfermagem os impedia de serem apropriados. A alimentação com PEG fornece o acesso mais valioso para nutrição em pacientes com um sistema gastrointestinal funcional. Sua alta eficiência, segurança e baixo custo sustentam a crescente popularidade em todo o mundo.

Palavras-chave: Gastrostomia, Alimentação por sonda, Nutrição enteral, Tubos de alimentação gástrica, Intubação gastrointestinal, Cuidados de enfermagem, Complicações. 


\section{Introducción}

Las gastrostomías endoscópicas percutánea (PEG), descritos por primera vez en 1980, han llegado a ser ampliamente utilizado para proporcionar soporte nutricional enteral para pacientes que son incapaces de ingerir alimentos sólidos o líquidos debido a muchos trastornos, a pesar de haber conservado las funciones de absorción y de la motilidad del tracto gastrointestinal. En estos casos, los tubos de PEG han surgido como una alternativa a la nutrición artificial parenteral y especialmente a sondas nasogástricas, para la administración de los alimentos directamente en el estómago (que se reconoce como la opción de alimentación más adecuada y fisiológica).

La colocación PEG es una técnica endoscópica que permite la colocación de un tubo flexible para crear una comunicación temporal o permanente entre la pared abdominal y la cavidad gástrica, asegurando el paso directo de los alimentos en el tracto digestivo del paciente.

Incluso cuando el uso de alimentación por tubo PEG no ha sido universalmente demostrado para disminuir los riesgos de neumonía por aspiración (Onur \& Guneysel, 2013) o la mortalidad a largo plazo, ni los resultados con respecto al mantenimiento de peso en comparación con la alimentación nasogástrica tubo en varios grupos de pacientes, la alimentación PEG se ha demostrado consistentemente que el método de alimentación con una menor probabilidad de fallo de la intervención, lo que sugiere el procedimiento endoscópico es más efectivo y segura de la alimentación por sonda nasogástrica, de acuerdo con una revisión sistemática Cochrane (Gomes, Lustosa, \& Matos, 2012).

Los tubos de PEG han reemplazado otras técnicas de gastrostomía y la radiológico-quirúrgicas como el método de elección para la alimentación a largo plazo de los pacientes que son incapaces de nutrición principal adecuada en la presencia de un funcionamiento gastrointestinal normal. Como resultado, el uso de PEG es reconocido como un procedimiento mínimamente invasivo que elimina la necesidad de anestesia general y requiere menos instrumentación, es por lo tanto una valiosa fuente de nutrición por la alimentación enteral en hogares de ancianos y entornos domiciliarios cuando el periodo de administración es espera que exceda de 4 semanas y la esperanza de vida de los pacientes supera los dos meses (Sartori \& Trevisani, 2006). Se ve favorecida por su simplicidad, utilidad, seguridad, facilidad de uso y bajo coste.

Este artículo pretende revisar la evidencia actual de las indicaciones y ventajas de la colocación del tubo PEG en variedad de entornos y condiciones patológicas. las técnicas de colocación y de gestión de procedimiento de tubos PEG también se explicarán y riesgos y complicaciones potenciales discutidos. Por último, se prestará atención de enfermería específica.

\section{Metodología}

Para el desarrollo de este proceso investigativo, se plantea como metodología la encaminada hacia una orientación científica particular que se encuentra determinada por la necesidad de indagar en forma precisa y coherente una situación, en tal sentido (Davila, 2015) define la metodología "como aquellos pasos previos que son seleccionados por el investigador para lograr resultados favorables que le ayuden a plantear nuevas ideas".(p.66)

Lo citado por el autor, lleva a entender que el desarrollo de la acción investigativa busca simplemente coordinar acciones enmarcadas en una revisión bibliográfica con el fin de complementar ideas previas relacionadas a la gastrostomía endoscópica percutánea a través de una revisión de literatura, para así finalmente elaborar un cuerpo de consideraciones generales que ayuden a ampliar el interés propuesto. 


\section{Tipo de Investigación}

Dentro de toda práctica investigativa, se precisan acciones de carácter metodológico mediante las cuales, se logra conocer y proyectar los eventos posibles que la determinan, así como las características que hacen del acto científico un proceso interactivo ajustado a una realidad posible de ser interpretada. En este sentido, se puede decir, que la presente investigación corresponde al tipo documental, definido por Castro (2016), "se ocupa del estudio de problemas planteados a nivel teórico, la información requerida para abordarlos se encuentra básicamente en materiales impresos, audiovisuales y /o electrónicos". (p.41).

En consideración a esta definición, la orientación metodológica permitió la oportunidad de cumplir con una serie de actividades inherentes a la revisión y lectura de diversos documentos donde se encontraron ideas explicitas relacionadas con los tópicos encargados de identificar a cada característica insertada en el estudio. Por lo tanto, se realizaron continuas interpretaciones con el claro propósito de revisar aquellas apreciaciones o investigaciones propuestas por diferentes investigadores relacionadas con el tema de interés, para luego dar la respectiva argumentación a los planteamientos, en función a las necesidades encontradas en la indagación.

\section{Fuentes Documentales}

El análisis correspondiente a las características que predomina en el tema seleccionado, llevan a incluir diferentes fuentes documentales encargadas de darle el respectivo apoyo y en ese sentido cumplir con la valoración de los hechos a fin de generar nuevos criterios que sirven de referencia a otros procesos investigativos. Para (CASTRO, 2016) las fuentes documentales incorporadas en la investigación documental o bibliográfica, "representa la suma de materiales sistemáticos que son revisados en forma rigurosa y profunda para llegar a un análisis del fenómeno".(p.41). Por lo tan- to, se procedió a cumplir con la realización de una lectura previa determinada para encontrar aquellos aspectos estrechamente vinculados con el tema, con el fin de explicar mediante un desarrollo las respectivas apreciaciones generales de importancia.

\section{Técnicas para la Recolección de la Infor- mación}

La conducción de la investigación para ser realizada en función a las particularidades que determinan a los estudios documentales, tiene como fin el desarrollo de un conjunto de acciones encargadas de llevar a la selección de técnicas estrechamente vinculadas con las características del estudio. En tal sentido, (Bolívar, 2015), refiere, que es "una técnica particular para aportar ayuda a los procedimientos de selección de las ideas primarias y secundarias". (p. 71).

Por ello, se procedió a la utilización del subrayado, resúmenes, fichaje, como parte básica para la revisión y selección de los documentos que presentan el contenido teórico. Es decir, que mediante la aplicación de estas técnicas se pudo llegar a recoger informaciones en cuanto a la revisión bibliográfica de los diversos elementos encargados de orientar el proceso de investigación. Tal como lo expresa, (Bolívar, 2015) "las técnicas documentales proporcionan las herramientas esenciales y determinantes para responder a los objetivos formulados y llegar a resultados efectivos" (p. 58). Es decir, para responder con eficiencia a las necesidades investigativas, se introdujeron como técnica de recolección el método inductivo, que hizo posible llevar a cabo una valoración de los hechos de forma particular para llegar a la explicación desde una visión general.

Asimismo, se emplearon las técnicas de análisis de información para la realización de la investigación que fue ejecutada bajo la dinámica de aplicar diversos elementos encargados de determinar el camino a recorrer por el estudio, según, (Bolívar, 2015) las técnicas de procesamiento de datos en 
los estudios documentales "son las encargadas de ofrecer al investigador la visión o pasos que debe cumplir durante su ejercicio, cada una de ellas debe estar en correspondencia con el nivel a emplear" (p. 123). Esto indica, que para llevar a cabo el procesamiento de los datos obtenidos una vez aplicado las técnicas seleccionadas, tales como: fichas de resumen, textual, registros descriptivos entre otros, los mismos se deben ajustar al nivel que ha sido seleccionado.

\section{Resultados}

\section{Indicaciones para gastrostomía endoscó- pica percutánea}

La opción para alimentar a un paciente a través de un tubo de PEG debe ser considerada en diferentes situaciones, tanto en el hospital y en casa. De hecho, varias condiciones crónicas pueden ser aliviados mediante la alimentación de enfermos con un tracto digestivo intacto a través de un tubo de PEG. Una reducción en la ingesta oral, generalmente debido a procesos neurodegenerativos representa la razón principal para la colocación PEG en hasta el 90\% de los casos. Sin embargo, la alimentación por sonda PEG en pacientes con demencia ha sido en gran medida controvertida: El amplio uso de estos dispositivos en situaciones de contraste insuficiencia nutricional oral con la falta de beneficios probados en pacientes con demencia avanzada, que no se demostró en una revisión sistemática que incluyó siete estudios de observación (Sampson \& Candy, 2012): No hubo evidencias de aumento de la supervivencia, mejora del estado nutricional o reducción de las úlceras por presión tasas de prevalencia en pacientes que reciben alimentación enteral por sonda. Por lo tanto, la decisión final para la colocación del tubo PEG en pacientes con demencia y otras enfermedades neurodegenerativas debe evaluarse entre el médico, familia y cuidadores, teniendo en cuenta las instrucciones anticipadas del paciente.
Además, una aspiración bronquial repetida de los alimentos, o la obstrucción derivada de la orofaringe, el cuello o los tumores esofágicos, están otras indicaciones comunes. La Tabla I incluye las mayoría de las indicaciones frecuentes para la colocación PEG, clasificar a los pacientes de acuerdo con la cronicidad de las enfermedades subyacentes y su capacidad de recuperación.

Un cuerpo creciente de literatura está documentando el valor potencial de la colocación de tubo PEG en el inicio del tratamiento en pacientes con lesión de cabeza y cuello puede, que están en mayor riesgo de desnutrición y disfagia (Lucendo Villarín \& Polo Araujo, 2015). En estos pacientes, la alimentación enteral se requiere a menudo en respuesta a la disfagia, odinofagia u otros efectos secundarios del tratamiento que conducen a la deshidratación y / o la pérdida de peso durante o después del tratamiento del cáncer. La mayoría de estudios publicados en la literatura apoyo nutricional generalmente comienzan por un tubo de PEG cuando esté indicado clínicamente en respuesta a un deterioro en la deglución o el estado nutricional. En contraste, algunos estudios han informado sobre el inicio de la alimentación enteral antes del tratamiento, que muestra que la colocación PEG profiláctica y la alimentación enteral temprana se asoció con una pérdida limitada de peso, lo que permite una nutrición e hidratación eficaz y segura del paciente durante la quimio radiación. Además, los pacientes que requieren la colocación del tubo PEG terapéutica en respuesta a la pérdida de peso significativa durante el tratamiento sufrieron mayor morbilidad que los pacientes que recibieron tubos $P E G$ profilácticamente (Cady, 2017).

Las pruebas que apoyan claramente la colocación temprana y el uso de un tubo de PEG en pacientes sometidos a tratamiento para el cáncer de cabeza y cuello es débil sin embargo, y los beneficios frente a riesgos no han sido definitivamente establecida. 
En la población pediátrica, la inserción de PEG para la nutrición enteral ha sido ampliamente aceptada, después de haber sido demostrado como una técnica eficaz y segura incluso en lactantes pequeños, y se asocia con una tasa aceptable de complicaciones (Fröhlich \& Richter, 2015). Una gama de la experiencia del clínico que demuestra una mejora en o mantenimiento de un estado nutricional adecuado en pacientes con una variedad de trastornos subyacentes (así como un alto nivel de aceptación por parte de los cuidadores), se ha reflejado en el aumento del número de condiciones médicas para que la alimentación PEG se indica en los niños. Estos incluyen no sólo los trastornos neurológicos, o malformaciones congénitas que conducen a la disfagia orofaríngea, sino también condiciones médicas y quirúrgicas que menoscabe una ingesta calórica adecuada.

El uso de la PEG en oncología pediátrica ha aumentado en los últimos años. En estas situaciones particulares de la alimentación temprana PEG, la colocación de PEG es capaz de revertir la pérdida de peso y representa una forma relativamente segura para prevenir la desnutrición en niños con cáncer, y por consiguiente podría desempeñar un papel en el resultado oncológico.

\section{Contraindicaciones para PEG}

Hay pocas contraindicaciones absolutas para la colocación de PEG, y éstos incluyen principalmente limitaciones técnicas como resultado de las particularidades anatómicas, tales como la falta de transiluminación con una incapacidad para acceder a la pared gástrica anterior, incluyendo interposición colónica y ascitis grave, coagulopatía avanzada no corregible, hipertensión portal con gástrica significativa várices que conduce al riesgo de hemorragia inasumibles; finalmente, la obstrucción de la faringe o del esófago que bloquea el paso del gastroscopio en el estómago evitará que una colocación de la sonda PEG. Las contraindicaciones relativas restantes son considerados
(Tabla II).

La cirugía abdominal previa actualmente no se considera una contraindicación para la colocación de la PEG, con estudios clínicos que demuestran que se puede colocar de forma segura en estos pacientes, con una alta tasa de éxito. La cirugía gástrica puede representar un desafío único para el endoscopista, con un $28 \%$ de los fallos de colocación registrados en un informe retrospectivo (Eleftheriadis \& Kotzampassi, 2011).

\section{Preparar al paciente para un PEG}

\section{Consentimiento informado}

El consentimiento informado debe ser obtenido de los pacientes o sus sustitutos legales que toman las decisiones de manera consensuada con los profesionales sanitarios. Los pacientes con demencia avanzada y disfagia generalmente se someten a la PEG, por lo que el consentimiento para un tratamiento en un paciente sin capacidad legal debe estar garantizado desde sustitutos legales nominados. La intención del consentimiento informado es para mejorar el cuidado del paciente, proporcionando ellos o su cuidador con la información completa sobre los beneficios y riesgos de la alimentación por sonda y los medicamentos antes de la inserción de PEG. 
Tabla 1. Las indicaciones para la colocación de una gastrostomía endoscópica percutánea

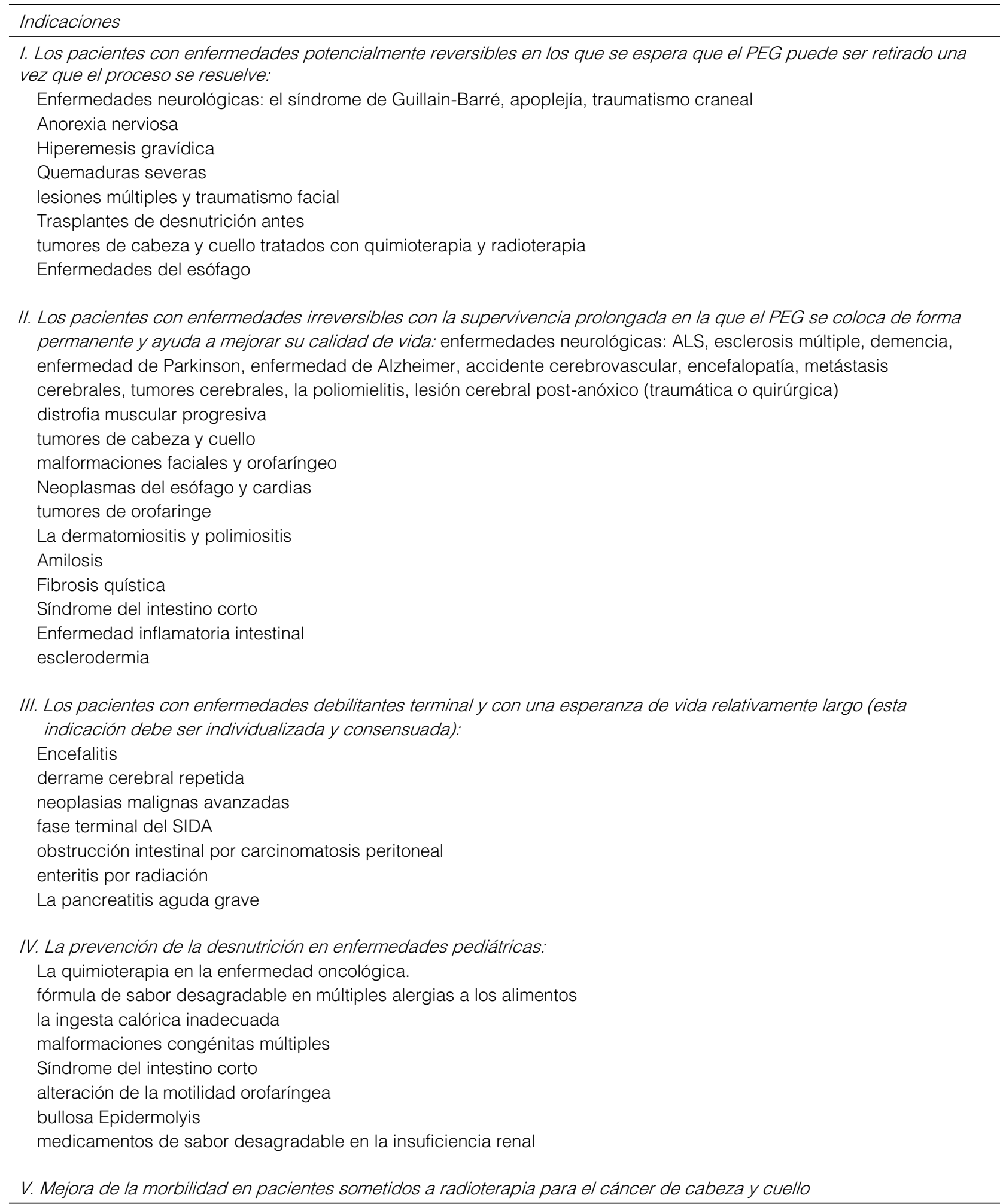

ALS: esclerosis lateral amiotrófica; SIDA: Síndrome de inmunodeficiencia adquirida.

Fuente: Autores 2020 
La prevención de la infección del estomago

Aunque PEG se considera un procedimiento relativamente de cirugía menor, que se asocia con complicaciones generales, entre las cuales infección de la herida es el problema común. La colocación de un tubo de PEG no se considera una técnica estéril y pacientes sometidos a ella son a menudo vulnerables de infección por una variedad de razones, incluyendo la vejez, comprometida ingesta nutricional, la inmunosupresión y la enfermedad subyacente tal como malignidad y diabetes. Las bacterias que colonizan el tracto nasofaringeal y digestivo superior pueden causar infección periostomal utilizando la técnica de tracción, una complicación que se describe con una frecuencia de hasta $32 \%$ sin profilaxis anti- biótica (Grant, 2003).

Una revisión sistemática con metaanálisis de ECA demostró una reducción significativa en la incidencia de infección periostomal cuando se administraron antibióticos profilácticos por vía intravenosa (agruparon OR 0,31, IC 95\%, 0,22 a 0,44). Los antibióticos usados más comúnmente para prevenir la infección periostomal se administran por vía intravenosa betalactamics, incluyendo coamoxoclav, cefotaxima, cefoxitina o cefazolina, antes de PEG. Un ECA reciente, sin embargo, la comparación de la administración de $20 \mathrm{ml}$ de solución de cotrimoxazol depositado en un catéter PEG recién insertado a la cefuroxima profilaxis administrada por vía intravenosa antes de PEG fue al menos tan eficaz en la prevención de infecciones de la herida (Blomberg \& Lagergren, 2010).

Tabla 2. Contraindicaciones para la colocación de una gastrostomía endoscópica percutánea

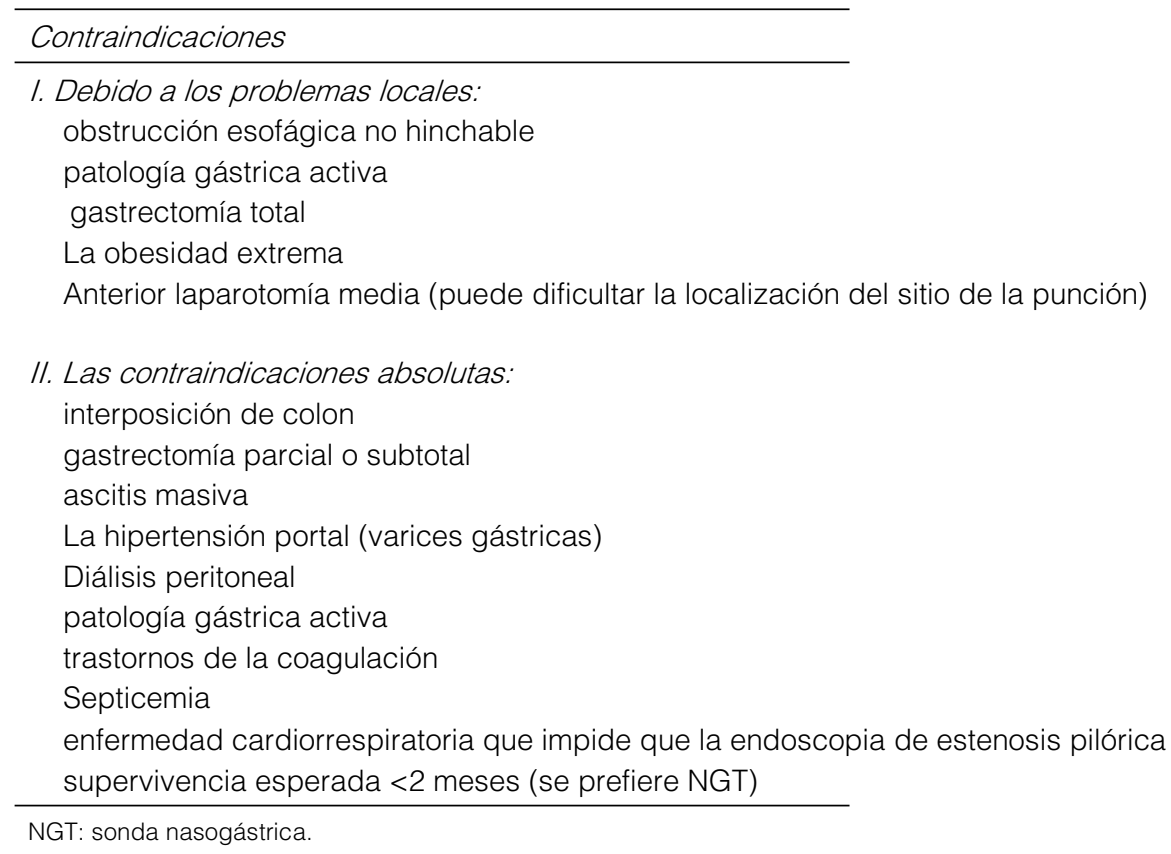

Fuente: Autores 2020 


\section{Materiales}

El dispositivo de PEG normalmente se comercializa como un kit, que incluye: jeringa y aguja, escalpelo, trocar, guías de hilo, el tubo y trampa. Además de este material, medicación para sedo analgesia y anestesia local debe proporcionar, junto con las herramientas para administrarlos y para aspirar las secreciones de la orofaringe, si es necesario.

\section{Técnica de colocación}

Para insertar un tubo de PEG por lo general requiere un equipo de 3 personas (generalmente 2 endoscopistas / gastroenterólogos y una enfermera). El paciente se coloca en posición supina, vigilados y oxígeno mediante una cánula nasal administrado. Después de la desinfección de la pared abdominal para crear un campo estéril, los pacientes deben someterse a un esófago-gastro-duodenoscopia completa (EGD), con la insuflación de dióxido de aire / carbono máximo para la extensión de la pared del estómago. El sitio exacto de la inserción de PEG se determina mediante transiluminación gastroscópico y palpación manual desde el exterior para la confirmación visualizada de la colocación apropiada en la parte inferior del estómago.

El sitio de inserción del tubo de PEG es idealmente en la línea mediana (línea alba) para evitar hematoma y las infecciones en los compartimentos de los músculos rectos. A continuación, una aguja estará entrando a través de la piel en el estómago en la ubicación donde el tubo PEG se va a colocar.

\section{La atención al paciente después de la co- locación PEG}

Se recomienda tomar reposo en cama durante al menos 6 horas después de la colocación y para el seguimiento de cerca todos los signos vitales, así como cualquier aparición de dolor abdominal, fiebre o sangrado gastrointestinal. Es aconsejable mantener una línea venosa periférica insertada durante al menos 6 horas en caso de que surjan complicaciones. Además, algunos analgesicos pueden ser necesaria durante los dos primeros días, especialmente en el caso de los niños (Heuschkel, Gottrand, \& Devarajan, 2014).

\section{La identificación de factores de riesgo de complicaciones}

Hay varios informes retrospectivos en aumento de la conciencia de los factores de riesgo de complicaciones relacionadas con el PEG, con el objetivo de disminuir los costos de las molestias del paciente y de la salud. Entre los factores de riesgo no modificables, la edad avanzada es reconocido como aumentar el riesgo de muerte después de la inserción de PEG en el 1\% / año; específicamente una edad de más de 75 años ha sido identificado como un factor predictivo de muerte temprana 1 mes después de la colocación PEG (OR = 2,49;\% Cl = 1,47 a 4,21) (Light \& Slezak, 1995). La malnutrición, expresa tanto como una disminución del índice de masa corporal y baja albúmina de suero los niveles, está asociado repetidamente con una alta mortalidad y alta tasa de complicaciones después de PEG, así como la presencia de comorbilidades. De hecho, los subroga proteína (CRP) niveles de $\mathrm{C}$ reactiva y los recuentos de leucocitos anormales altos estaban relacionados con un aumento en la tasa de mortalidad temprana. La coexistencia de insuficiencia cardíaca congestiva, insuficiencia renal, infección del tracto urinario, la aspiración anterior, enfermedad pulmonar crónica, coagulopatía, trastornos de la circulación, cáncer de meta-estática, y la enfermedad hepática eran todos ellos fuertemente asociado con un aumento de la mortalidad. La suma de varios factores de riesgo en el mismo paciente también aumenta en gran medida la probabilidad de muerte temprana después de la inserción de un tubo de PEG.

El riesgo de complicaciones, incluyendo la muerte, siempre debe evaluarse individual- 
mente en cada paciente sometido a inserciones de tubos de PEG; Sin embargo, hay que tener siempre en cuenta que la alimentación enteral es superior a la alimentación parenteral en el paciente nutricionalmente empobrecido, y la alimentación PEG sigue siendo el método más seguro, más fácil y menos caro para la alimentación por sonda para una amplia gama de pacientes gravemente comprometidas. De hecho, la indicación para PEG se asocia fuertemente en sí con la mortalidad.

La colocación del tubo de PEG por un endoscopista sin experiencia ha sido identificado como un modificables factores de riesgo relacionados con complicaciones tempranas. Además, la inserción del parachoques interna de un tubo de PEG en el cuerpo superior del estómago también era un riesgo significativo de complicaciones tempranas y tardías.

\section{Conclusiones}

La alimentación a través de un tubo de PEG es el método deseable para alimentar pacientes con disfagia o en aquellos pacientes que no pueden alimentarse por vía oral, pero tienen un sistema digestivo funciona. La técnica se ha convertido en tareas más extendida a su simplicidad, seguridad y bajo costo. Por conducta adecuada, se requiere una formación específica para los profesionales responsables de estos procedimientos, ya su vez, a partir de ellos para

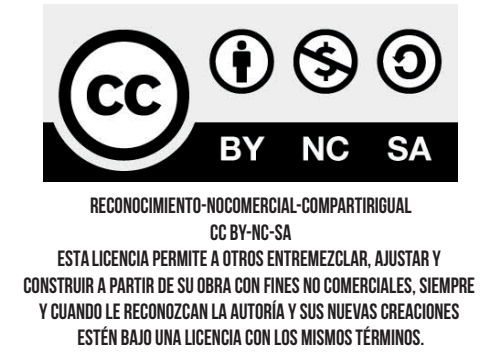

proporcionar formación e información a otros profesionales y cuidadores implicados en la atención al paciente. La administración de la atención adecuada a la medida y personalizados para cada caso, la adopción de estrategias de prevención, identificación y tratamiento de las complicaciones tempranas va a maximizar los resultados de seguridad y eficacia para los pacientes.

\section{Bibliografía}

Blomberg, J., \& Lagergren, P. (2010). Novel approach to antibiotic prophylaxis in percutaneous endoscopic gas-trostomy (PEG): Randomised controlled trial. BMJ , 241.

Bolívar, J. (2015). Investigación Documental. México. Pax.

Cady, J. (2017). Nutritional support during radiotherapy for head and neck cancer: The role of prophylactic feeding tube placement. Clin J Oncol Nurs , 875-80.

Castro, J. (2016). Técnicas Documentales. México. Limusa.

Davila, A. (2015). Concepto de terminos cientificos. Caracas: Oasis.

Eleftheriadis, E., \& Kotzampassi, K. (2011). Percutaneous endoscopic gastrostomy after abdominal surgery. . Surg Endosc, 213-6.

Fröhlich, T., \& Richter, M. (2015). Review article: Percutaneous endoscopic gastrostomy in infants and children. Aliment Pharmacol Ther, 788-801.

Gomes, C. J., Lustosa, S., \& Matos, D. (2012). Percutaneous endoscopic gastrostomy versus nasogastric tube feeding for adults with swallowing disturbances. . Cochrane Data-base Syst Rev.

Grant, J. (2003). Percutaneous endoscopic gastrostomy. Initial placement by single endoscopic technique and long-term follow-up. .Ann Surg, 168-74.

\section{CITAR ESTE ARTICULO:}

Crespo Zamora, M., Parrales Vidal, I., Romoleroux Uquillas, G., \& Coello Vergara, J. (2020). Gastrostomía endoscópica percutánea indicaciones clínicas. RECIMUNDO, 4(1(Esp)), 219-228. doi:10.26820/recimundo/4.(1).esp.marZo.2020.219-228 